\title{
ÍNDICES DE GERAÇÃO DE RESÍDUOS SÓLIDOS EM RESTAURANTE INDUSTRIAL DE GRANDE PORTE
}

\section{SOLID WASTES GENERATION INDEX IN LARGE INDUSTRIAL RESTAURANT}

\author{
Maria Lúcia Ribeiro \\ Docente do Programa de Mestrado em Desenvolvimento Regional e Meio Ambiente pelo Centro Universitário de Araraquara - UNIARA. \\ Araraquara, SP, Brasil. \\ Email: mlucia@iq.unesp.br
}

Rita de Cássia Reis Carvalho

Mestre em Desenvolvimento Regional e Meio Ambiente pelo Centro Universitário de Araraquara - UNIARA.

Araraquara, SP, Brasil.

Email: rikare@bol.com.br

\section{Marcus César Avezum Alves de Castro}

Docente do Programa de Mestrado em Desenvolvimento Regional e Meio Ambiente pelo Centro Universitário de Araraquara - UNIARA. Araraquara, SP, Brasil.

Email: mestrado@uniara.com.br

\section{Marina Gulo Alcorinte}

Bolsista de Apoio Técnico da Fundação Nacional de Desenvolvimento do Ensino Superior Particular (FUNADESP) - Centro Universitário de Araraquara - UNIARA.

Araraquara, SP, Brasil.

Email: marina.alcorinte@gmail.com

\section{Danielle dos Santos Araújo}

Bolsista de Apoio Técnico da Fundação Nacional de Desenvolvimento do Ensino Superior Particular (FUNADESP) - Centro Universitário de Araraquara - UNIARA.

Araraquara, SP, Brasil.

Email: dsa.danielle@bol.com.br

\section{Larissa Fernanda Cazeiro}

Bolsista de Apoio Técnico da Fundação Nacional de Desenvolvimento do Ensino Superior Particular (FUNADESP) - Centro Universitário de Araraquara - UNIARA.

Araraquara, SP, Brasil.

Email: larissa.cazeiro@hotmail.com

\section{RESUMO}

Considerando a importância econômica, social e ambiental que $o$ setor dos restaurantes industriais $(\mathrm{RI})$ vem alcançando no mundo contemporâneo, este estudo objetivou quantificar resíduos gerados em um RI de grande porte do estado de São Paulo. As coletas foram realizadas durante 12 meses em 2009, nos três turnos de distribuição das refeições. A determinação da geração de resíduos/refeição foi realizada nas quatro fontes geradoras: retorno de bandejas, pré-preparo e preparo, sobra limpa e recicláveis. Foram gerados, no total, $29.356 \mathrm{~kg}$ de resíduos, correspondendo a $72,5 \%$ de matéria orgânica e $27,4 \%$ de recicláveis. O índice médio de geração de resíduos foi de $59,3 \mathrm{~g} /$ refeição, destacando-se a devolução com a maior contribuição (19,6g/refeição) e a etapa de sobra limpa como menor produtora de resíduos (12,5g/refeição), indicando um protocolo de rotina eficaz. A notória quantidade de resíduos orgânicos gerados deve ser minimizada por práticas como compostagem, utilização de alimentos que produzem menos resíduos ou pré-processados, bem como investir na educação ambiental de usuários e funcionários.

Palavras-chave: Gestão de resíduos. Índice de geração de resíduos. Restaurantes industriais. Resíduos orgânicos. Resíduos sólidos urbanos.

\begin{abstract}
Considering the economic, social and environmental importance of the industrial restaurants (IR) in the current world, this study aimed to quantify wastes generated in a large IR of São Paulo's state. The collect was performed during 12 months in 2009, at the three meals distribution shifts. The determination of waste generation/meal was conduct in the four generating sources: trays return, prepreparation and preparation, clean waste and recyclables. A total of $29.356 \mathrm{~kg}$ of waste was generate, corresponding to $72.5 \%$ of organic material and 27.4 of recyclables. The average rate of waste generation was $59.3 \mathrm{~g} / \mathrm{meal}$, highlighting the return with the largest contribution $(19.6 \mathrm{~g} / \mathrm{meal})$ and the clean waste step as the minor wastes producer $(12.5 \mathrm{~g} / \mathrm{meal})$ indicating an effective routine protocol. The amount notorious of organic waste generated should be minimize by practices such as composting, use of foods that produce less waste or pre-processed as well as invest in the environmental education of users and staff.
\end{abstract}

Keywords: Waste management. Index of waste generation. Industrial restaurants. Organic wastes. Solid urban wastes. 


\section{INTRODUÇÃO}

A gestão de resíduos sólidos urbanos (RSU) no Brasil apresenta-se como uma problemática ambiental crescente, que acompanha a dinâmica populacional e tecnológica, caracterizada pela geração excessiva, desperdício e mau gerenciamento dos resíduos (CARNEIRO et al., 2010).

A Política Nacional de Resíduos Sólidos, instituída pelo Decreto 7404/2010, define resíduos sólidos como "resíduos nos estados sólido e semissólido, que resultam de atividades de origem urbana, industrial, de serviços de saúde, rural, especial ou diferenciada". Já a Associação Brasileira de Normas Técnicas, por meio da Norma Brasileira 10004:2004, define resíduos sólidos como:

"Resíduos nos estados sólido e semissólido, que resultam de atividades da comunidade de origem: industrial, doméstica, hospitalar, comercial, agrícola, de serviços e de varrição, os lodos provenientes de sistemas de tratamento de água, aqueles gerados em equipamentos e instalações de controle de poluição, bem como determinados líquidos cujas particularidades tornem inviável seu lançamento na rede pública de esgotos ou corpos d'água, ou exijam para isso soluções técnicas e economicamente inviáveis em face à melhor tecnologia disponível."

Segundo a Associação Brasileira de Empresas de Limpeza Pública e Resíduos Especiais (ABRELPE), a geração de RSU cresceu 1,3\% de 2011 a 2012, ficando à frente da taxa de crescimento populacional do mesmo período. Ainda, segundo dados da pesquisa, a matéria orgânica correspondeu a $51,4 \%$ dos resíduos totais gerados (Tabela 1 ).

Tabela 1: Porcentagem de geração dos principais RSU em 2012.

\begin{tabular}{lcc}
\hline \multicolumn{1}{c}{ Material } & Participação (\%) & Quantidade (t/ano) \\
\hline Metais & 2,9 & 1.640 .294 \\
Papel, papelão e TetraPak & 13,1 & 7.409 .603 \\
Plástico & 13,5 & 7.635 .851 \\
Vidro & 2,4 & 1.357 .484 \\
Matéria Orgânica & 51,4 & 29.072 .794 \\
Outros & 16,7 & 9.445 .830 \\
\hline Total & $\mathbf{1 0 0 , 0}$ & $\mathbf{5 6 . 5 6 1 . 8 5 6}$ \\
\hline
\end{tabular}

Fonte: ABRELPE, 2012.

Apesar dos resíduos serem compostos por mais de $50 \%$ por matéria orgânica, os principais esforços no gerenciamento têm ocorrido no sentido da reciclagem de embalagens. Programas de coleta seletiva são direcionados para materiais como plásticos, papel, metais e vidro. Observa-se pouca ou nenhuma iniciativa de reaproveitamento da matéria orgânica presente nos resíduos domiciliares. Entretanto, a segregação da fração orgânica na fonte de geração, apresenta boas características para ser utilizada no processo de compostagem. (CASTRO, 2013).

Diante deste cenário, as empresas que fornecem refeições coletivas têm papel fundamental na geração desses resíduos, uma vez que o setor terceirizado de refeições coletivas no Brasil forneceu cerca de 10,5 milhões de refeições/dia no ano de 2011 e, estima-se que esse valor alcance 12,5 milhões em 2014 
(ABERC, 2008), consumindo toneladas de alimento/dia e gerando RSU com a mesma intensidade. Dessa forma, os restaurantes industriais (RI), que podem ser caracterizados como espaços voltados para o preparo e fornecimento de refeições coletivas saudáveis, seguras e equilibradas em nutrientes para populações específicas, fazem parte da geração de RSU primariamente orgânicos, estando sujeitos à elaboração do plano de gerenciamento de resíduos sólidos (MONTE et al., 2004; TRANCOSO, 2004).

Normalmente, o número de usuários que frequentarão o RI é previamente definido, em decorrência de contratos firmados entre as empresas fornecedoras e consumidoras. Dentre os procedimentos operacionais seguidos em um RI, destaca-se a estrutura física e a divisão em setores, em que cada um gera resíduos ao final da sua atividade diária. Periodicamente acompanhada, a geração de resíduos através das sobras dos alimentos é indicadora de consumo inadequado, representando importância econômica para as empresas deste setor. Entretanto, o fator econômico não é o único considerado, pois minimizar o impacto ao meio ambiente nas etapas de produção e distribuição das refeições também compreende um compromisso das empresas e está baseado em normativas.

A literatura vem demonstrando a preocupação com o impacto ambiental que as atividades dos restaurantes de alimentação coletiva podem causar e atribuem a minimização desse problema à educação ambiental (BILCK et al., 2009; CARNEIRO et al., 2010; CORRÊA; LANGE, 2010; VIDMANTAS et al., 2010; LAFUENTE JUNIOR, 2012). Além disso, Peruchin et al. (2013) apontam que a falta de infraestrutura adequada prejudica o gerenciamento correto dos resíduos, bem como Thiagarajah e Getty (2013) demonstram que os métodos de servir as refeições podem contribuir para a diminuição dos resíduos gerados. Embora Lopes e Fonseca (2013) indiquem que o restaurante por eles estudado apresentou consciência ambiental em relação aos impactos que o descarte inadequado dos resíduos pode causar, tem-se lacunas importantes no descarte dos descartáveis e o resto ingesta.

Considerando a importância econômica, social e ambiental que o setor dos restaurantes industriais vem alcançando no mundo contemporâneo, este estudo tem como finalidade quantificar os resíduos gerados em um RI de uma empresa, avaliando as possibilidades de minimizar o impacto ao meio ambiente nas etapas de geração de resíduos no processo de produção e distribuição das refeições.

\section{PROCEDIMENTOS METODOLÓGICOS}

\section{A estrutura do RI em estudo}

Localizado na região central do estado de São Paulo, o RI estudado atende a aproximadamente 2.000 usuários/dia (distribuídos entre almoço, jantar e ceia) no sistema autoatendimento, com serviços esporádicos de preparo e distribuição de lanches, cafés e outros eventos. O restaurante é administrado por uma empresa terceirizada, a qual define o padrão de cardápio, os serviços oferecidos e todas as responsabilidades que envolvem o processo de fabricação e distribuição dos serviços.

O quadro de funcionários do RI é estabelecido de acordo com o número de serviços prestados e de usuários do restaurante, sendo composto no momento da pesquisa por 47 funcionários, treinados e integrados às normas de boas práticas das empresas contratada e contratante, dentre elas, política de meio ambiente. 


\section{Quantificação dos resíduos gerados no RI}

Os resíduos gerados foram coletados durante um período de 12 meses no ano de 2009, nos três turnos de distribuição das refeições, obedecendo ao seguinte procedimento:

A pesagem dos resíduos foi realizada por segregação nas quatro fontes geradoras: retorno de bandejas; pré-preparo e preparo, sobra limpa e recicláveis, utilizando balança plataforma digital, com capacidade para $200 \mathrm{~kg}$, nos diferentes setores do restaurante como visualizado no Quadro 1.

Quadro 1: Caracterização dos resíduos coletados nos diferentes setores do RI.

\begin{tabular}{|l|l|}
\hline \multicolumn{1}{|c|}{ Setores } & \multicolumn{1}{c|}{ Caracterização } \\
\hline Retorno de bandejas & Resíduos provenientes dos pratos dos usuários e cascas de frutas consumidas; \\
\hline Pré-preparo e Preparo & $\begin{array}{l}\text { Resíduos gerados no preparo do cardápio diário, nas áreas de pré-preparo e preparo de } \\
\text { saladas, sobremesas, cocção e açougue: folhas, cascas, talos de legumes e frutas que } \\
\text { não serão utilizados nas preparaçães, restos dos recipientes usados para coç̧ão e } \\
\text { também resíduos provenientes de sobras na distribuição e que não poderão ser } \\
\text { reaproveitados. }\end{array}$ \\
\hline Sobra limpa & $\begin{array}{l}\text { Resíduos gerados no preparo do cardápio do dia, porém não utilizados ou distribuídos } \\
\text { ao usuário. Corresponde à produção da refeição que não foi servida, pois o prazo de } \\
\text { validade excedeu o período para uso, de acordo com critérios de utilização de alimentos } \\
\text { processados no dia de consumo ou preparação em excesso. }\end{array}$ \\
\hline Recicláveis & $\begin{array}{l}\text { Resíduos gerados na segregação de recicláveis, como guardanapos de papel usados, } \\
\text { embalagens de refrigerantes e copos descartáveis utilizados no consumo de } \\
\text { sobremesas e sucos; e embalagens provenientes do estoque do RI (papelão e plástico); }\end{array}$ \\
\hline
\end{tabular}

Fonte: Elaborado pelos autores, 2014.

\section{Análise dos dados obtidos}

Os dados obtidos foram organizados, categorizados e analisados para avaliar qualitativa e quantitativamente os resíduos gerados nas atividades produtivas do RI e no descarte pelos usuários, contemplando: resíduos gerados nos 12 meses de 2009 (registro mensal: peso em kg); resíduos gerados em cada fonte geradora do RI (registro mensal: peso em $\mathrm{kg}$ ); índice de geração de resíduos por refeição (gramas/refeição). 
RESULTADOS E DISCUSSÃO

Perfil dos resíduos gerados mensalmente em cada etapa em 2009

O perfil de resíduos gerados em 2009 no restaurante estudado, referente a 494.822 refeições, está apresentado na Tabela 2. Foram gerados, no total, $29.356 \mathrm{~kg}$ de resíduos, correspondendo a 72,5\% (21.287 kg) de matéria orgânica e 27,4\% (8.069kg) de recicláveis.

Tabela 2: Dados quantitativos da geração de resíduos sólidos em cada etapa do RI em 2009.

\begin{tabular}{|c|c|c|c|c|c|}
\hline \multirow{2}{*}{ Mês } & \multirow{2}{*}{$\begin{array}{c}\text { Número de refeições } \\
\text { servidas }\end{array}$} & \multicolumn{3}{|c|}{ Matéria orgânica (kg) } & \multirow{2}{*}{$\begin{array}{l}\text { Recicláveis } \\
\text { (kg) }\end{array}$} \\
\hline & & Devolução & $\begin{array}{l}\text { Pré-preparo e } \\
\text { preparo }\end{array}$ & Sobra limpa & \\
\hline Janeiro & 42.796 & 937 & 498 & $\left({ }^{*}\right)$ & 805 \\
\hline Fevereiro & 42.964 & 958 & 503 & $\left({ }^{*}\right)$ & 799 \\
\hline Março & 43.938 & 848 & 425 & 520 & 705 \\
\hline Abril & 41.718 & 798 & 408 & 550 & 689 \\
\hline Maio & 40.997 & 752 & 396 & 492 & 529 \\
\hline Junho & 39.906 & 768 & 408 & 503 & 521 \\
\hline Julho & 37.102 & 608 & 328 & 410 & 438 \\
\hline Agosto & 40.580 & 687 & 523 & 525 & 620 \\
\hline Setembro & 40.835 & 852 & 715 & 580 & 722 \\
\hline Outubro & 41.888 & 823 & 719 & 532 & 730 \\
\hline Novembro & 41.146 & 848 & 731 & 530 & 750 \\
\hline Dezembro & 40.952 & 863 & 759 & 490 & 761 \\
\hline Total & 494.822 & 9.742 & 6.413 & 5.132 & 8.069 \\
\hline
\end{tabular}

$\left(^{\star}\right)$ Nos meses de janeiro e fevereiro não foi contabilizada a geração de resíduos de sobra limpa.

Fonte: Elaborada pelos autores, 2014.

$\mathrm{Na}$ atividade relacionada ao retorno de bandejas, verificou-se que o hábito do usuário tem influência direta nos resíduos gerados, principalmente em relação à aceitação do cardápio pelo usuário: pratos de menor aceitação geram maior quantidade de resíduos na devolução. Nota-se, também, a redução do número de refeições servidas no mês de julho (férias), corroborando para a diminuição da geração de resíduos na devolução das bandejas. Nesta etapa, o valor médio de resíduos produzidos, em $\mathrm{kg}$, foi de 811,8, apresentando desvio padrão de 98,5 e coeficiente de variação de 12,1\% (Tabela 3).

Tabela 3: Análises estatísticas sobre os dados quantitativos da geração de resíduos no RI em 2009.

\begin{tabular}{|c|c|c|c|c|c|}
\hline $\begin{array}{c}\text { Setores do } \\
\text { RI }\end{array}$ & Valores totais (2009) & Média $(\overline{\mathbf{x}})$ & $\begin{array}{l}\text { Desvio padrão } \\
\text { (S) }\end{array}$ & $\begin{array}{l}\text { Coeficiente de } \\
\text { variação }(\%)\end{array}$ & $\begin{array}{l}\text { Amplitude } \\
\text { (A) }\end{array}$ \\
\hline Devolução & 9.742 & 811,80 & 98,50 & 12,10 & 350 \\
\hline Pré-preparo e preparo & 6.413 & 534,40 & 154,90 & 28,90 & 431 \\
\hline Sobra limpa & 5.132 & 513,20 & 45,20 & 8,80 & 170 \\
\hline Recicláveis & 8.069 & 672,40 & 119,00 & 17,70 & 367 \\
\hline Refeições servidas & 494.822 & 41.235 & $1.731,40$ & 4,20 & 6.836 \\
\hline
\end{tabular}

Fonte: Elaborada pelos autores. 
O aumento de resíduos gerados na etapa de pré-preparo e preparo a partir do mês de agosto foi decorrente da utilização de materiais in natura para preparar saladas e pratos de acompanhamento, como batata, cenoura, couve, chuchu, beterraba, mandioca e outros alimentos que compõem o cardápio, os quais nos meses anteriores eram adquiridos de forma pré-processada (desprovidos de cascas e cortados em forma de cubos, palitos ou ralados). Entretanto é importante ressaltar que a geração de resíduo no pré-preparo e preparo é influenciada pelo treinamento dos funcionários responsáveis pela higiene, preparo e finalização dos alimentos. Com base nos dados observa-se também significativa variação da quantidade de resíduos gerados nas atividades do pré-preparo dos alimentos (desvio padrão: 154,9; coeficiente de variação: 28,9\%). Tal fato pode ser justificado pela prática específica requerida para cada alimento durante o seu preparo como, por exemplo, a retirada de cascas. Outro aspecto é variação na qualidade de hortifrúti recebidos, os quais podem demandar a seleção e descarte de produtos que não atendam ao padrão de qualidade.

$\mathrm{Na}$ etapa sobra limpa, pode-se observar que nos meses de janeiro e fevereiro o controle da geração de resíduos não era realizado. Considerando os meses de março a dezembro, essa etapa foi a de menor geração (A: $170 \mathrm{~kg}$ ) e o baixo valor do coeficiente de variação da sobra limpa $(8,8)$, comparado com as demais etapas, sinaliza para uma rotina (padrão) já bem estabelecida no planejamento da quantidade de alimento a ser servido, apesar da variação do número de refeições servidas.

A geração de resíduos recicláveis obteve a maior variação dos setores, destacando-se o mês de julho, o qual apresentou menor valor de geração. A média dos resíduos gerados nesta etapa foi de $672,4 \mathrm{~kg}$, ficando abaixo apenas da etapa de devolução, com coeficiente de variação de $17,7 \%$. A separação e quantificação dos resíduos da fonte geradora de recicláveis não é preocupação destacada na literatura, que tem centrado a discussão na determinação da quantidade de resíduos e no uso de copos plásticos para o consumo de água e suco, sugerindo uso de copos individuais não descartáveis aos clientes com o intuito de minimizar os resíduos (SIQUEIRA, 2002). Este enfoque pode ser compreendido em função dos resíduos orgânicos compreenderem a maior fonte de resíduos de um restaurante e da possibilidade de reciclagem de alguns tipos de descartáveis. Outro aspecto considerado é o uso de copos descartáveis para servir determinadas sobremesas, fato que onera a geração de recicláveis.

Finalmente, de maneira geral, como afirmado por Sousa et al. (2012), o desperdício e a geração de resíduos sólidos dentro das unidades de refeições coletivas estão relacionados a fatores como planejamento, frequência e preferência dos usuários, bem como ao treinamento de funcionários.

\section{Índice de geração de resíduos em cada etapa por refeição em 2009}

A determinação da geração de resíduos em gramas para cada usuário do RI foi realizada quantitativa e qualitativamente. Os índices globais mensais mantiveram-se com uma variação de $22,2 \mathrm{~g}$ referente aos meses coletados (Tabela 4). O índice médio, correspondente ao período anual, foi estimado a partir dos mensais, permitindo ser calculado um percentual em cada uma das etapas do processo do RI: devolução, pré-preparo e preparo, sobra limpa e recicláveis (Tabela 5). 
Tabela 4: Índices de geração de resíduos por refeição servida global e por setor no ano de 2009.

\begin{tabular}{|c|c|c|c|c|c|}
\hline \multirow[t]{2}{*}{ Mês } & \multirow{2}{*}{$\begin{array}{c}\text { Índices } \\
\text { globais } \\
\text { (g/refeição) }\end{array}$} & \multicolumn{3}{|c|}{$\begin{array}{l}\text { Índices por setor } \\
\text { (g/refeição) }\end{array}$} & \multirow{2}{*}{$\begin{array}{l}\text { Recicláveis } \\
\text { (g/refeição) }\end{array}$} \\
\hline & & Devolução & Pré-preparo e preparo & Sobra limpa & \\
\hline Janeiro & 52,3 & 21,9 & 11,6 & $\left({ }^{*}\right)$ & 18,8 \\
\hline Fevereiro & 52,6 & 22,3 & 11,7 & $\left({ }^{*}\right)$ & 18,6 \\
\hline Março & 56,8 & 19,3 & 9,7 & 11,8 & 16,0 \\
\hline Abril & 58,6 & 19,1 & 9,8 & 13,2 & 16,5 \\
\hline Maio & 52,9 & 18,3 & 9,7 & 12,0 & 12,9 \\
\hline Junho & 55,1 & 19,2 & 10,2 & 12,6 & 13,1 \\
\hline Julho & 48,1 & 16,4 & 8,8 & 11,0 & 11,8 \\
\hline Agosto & 58,0 & 16,9 & 12,9 & 12,9 & 15,3 \\
\hline Setembro & 70,3 & 20,9 & 17,5 & 14,2 & 17,7 \\
\hline Outubro & 66,9 & 19,6 & 17,2 & 12,7 & 17,4 \\
\hline Novembro & 69,5 & 20,6 & 17,8 & 12,9 & 18,2 \\
\hline Dezembro & 70,2 & 21,1 & 18,5 & 11,9 & 18,6 \\
\hline Índices médios & 59,3 & 19,6 & 12,9 & $12,5^{*}$ & 16,3 \\
\hline
\end{tabular}

$\left({ }^{*}\right)$ Nos meses de janeiro e fevereiro não foi contabilizada a geração de resíduos de sobra limpa. Fonte: Elaborada pelos autores.

Tabela 5: Análises estatísticas sobre índices de geração de resíduos por refeição no RI em 2009.

\begin{tabular}{lccccc}
\hline \multicolumn{1}{c}{$\begin{array}{c}\text { Setores do } \\
\text { RI }\end{array}$} & $\begin{array}{c}\text { Índices médios } \\
\text { (g/ref.) }\end{array}$ & $\begin{array}{c}\text { Frequência } \\
\text { percentual } \\
(\mathbf{\%})\end{array}$ & $\begin{array}{c}\text { Desvio } \\
\text { padrão } \\
\text { (S) }\end{array}$ & $\begin{array}{c}\text { Coeficiente de } \\
\text { variação (\%) }\end{array}$ & $\begin{array}{c}\text { Amplitude } \\
\text { (A) }\end{array}$ \\
\hline Devolução & 19,6 & 33,1 & 1,8 & 9,3 & 5,9 \\
Pré-preparo e preparo & 12,9 & 21,8 & 3,7 & 28,7 & 9,7 \\
Sobra limpa & 12,5 & $21,1^{*}$ & 0,9 & 6,9 & 14,2 \\
Recicláveis & 16,2 & 27,4 & 2,5 & 15,2 & 7,0 \\
\hline Índice global & 59,3 & 100,0 & 7,9 & 13,3 & 22,2
\end{tabular}

$\left(^{\star}\right)$ Nos meses de janeiro e fevereiro não foi contabilizada a geração de resíduos de sobra limpa.

Fonte: Elaborada pelos autores.

O índice médio de resíduos gerados no RI no ano de 2009 foi de 59,3g/refeição, em que 19,6g corresponderam à média do retorno das bandejas, $12,9 \mathrm{~g}$ ao pré-preparo e preparo, $12,5 \mathrm{~g}$ à sobra limpa e $16,2 \mathrm{~g}$ aos resíduos recicláveis. Dessa forma, foram determinadas as participações que cada etapa teve na geração total dos resíduos, destacando-se a devolução com a maior contribuição, seguida dos recicláveis, pré-preparo e preparo e, finalmente, a sobra limpa, como a menor produtora de resíduos. No trabalho de Picciafuoco (2013) o índice médio de geração de resíduos em um RU foi de aproximadamente 124g/refeição, sendo o setor de devolução o de maior geração, com $49 \%$ do total de resíduos gerados.

Em um estudo realizado por Souza et al. (2010) os restos de alimentos das bandejas representam $65 \%$ dos resíduos gerados em um restaurante universitário. No estudo atual, o valor encontrado foi de $33,1 \%$ na coleta do período anual. Picciafuoco (2013) investigou que $49 \%$ da produção total de resíduos durante um período de 10 dias correspondeu à etapa de devolução. Augustini et al. (2008), demonstram que, em sua pesquisa, o resto das bandejas por usuários manteve-se entre 40 e $90 \mathrm{~g}$, valores significativamente maiores dos encontrados neste estudo. Ainda, em Lafuente Junior (2012) os alimentos provenientes da devolução corresponderam a $32,86 \%$, sendo o resíduo de maior participação na geração total durante o período de um 
mês, indicando similaridade com os valores encontrados neste trabalho. Esses dados corroboram que o hábito do usuário é um fator que, embora variável, representa desperdício.

A etapa de pré-preparo e preparo foi responsável por $21,8 \%$ da geração total de resíduos no RI, com uma média de 12,9g produzidas por refeição. Picciafuoco (2013) constatou que $36 \%$ da geração total dos resíduos em um restaurante universitário corresponderam à etapa de preparo. Carneiro et al. (2010), observam que aproximadamente $90 \%$ dos resíduos sólidos gerados durante um mês em um restaurante universitário eram compostos de matéria orgânica, da qual $64 \%$ correspondeu ao preparo e pré-preparo e $36 \%$ foram provenientes do rejeito do refeitório (devolução). No presente trabalho, a etapa de pré-preparo e preparo correspondeu a 30,1\% da geração de resíduos orgânicos, ao passo que a devolução contribuiu com $45,8 \%$. A grande variação dos dados apresentados pode estar associada a procedimentos operacionais de cada estabelecimento e às metodologias utilizadas para contabilizar os materiais em cada setor.

A falta de gestão ou gestão inadequada e o desperdício nos restaurantes são os principais fatores descritos por Souza et al. (2010), quando analisou um restaurante universitário do Rio Grande do Sul, demonstrando que a matéria orgânica foi responsável por 85 e $78 \%$ do total de resíduos gerados em dois períodos coletados. Ainda, em dois outros estudos referente à geração de resíduos sólidos em restaurantes universitários, no Campus I da Universidade Estadual da Paraíba (COSTA et al., 2004) e no Campus de São Carlos da USP (MENEZES; SANTOS; LEME, 2002), os valores percentuais de orgânicos corresponderam a 97,21 e $74,6 \%$, respectivamente Neste estudo, a matéria orgânica produzida correspondeu a 72,5\%, ficando abaixo dos valores encontrados na literatura.

Assim como Picciafuoco et al. (2014), outros estudos referentes a RI ou restaurantes universitários (CARNEIRO et al., 2010; CORREAA; LANGE, 2010; SOUZA et al., 2010; LAFUENTE JUNIOR, 2012; PERUCHIN et al., 2013) propõem o tratamento dos resíduos orgânicos por meio da compostagem, a qual consiste na biodecomposição da matéria orgânica em material estável que pode ser aplicado ao solo. Porém, a qualidade desse material sofre influência de vários fatores, sendo o principal, a segregação dos tipos de resíduos nas fontes geradoras.

Para as sobras dos alimentos produzidos e não distribuídos, a literatura admite como aceitáveis percentuais de até $3 \%$ da produção ou de 7 a 25g de resíduo por usuário (VAZ, 2006 apud AUGUSTINI et al., 2008). Dessa forma, a média de 12,5g/refeição visualizada, encontra-se dentro dos padrões da literatura, bem como o percentual de toda a produção (1\%). No estudo de Lafuente Junior (2012), a sobra limpa foi a segunda etapa de maior geração de resíduos, com 20,94\%. No trabalho atual, investigou-se que embora a sobra limpa fosse a etapa de menor geração, a porcentagem de participação do resíduo no total assemelhase aos valores encontrados na literatura $(21,1 \%)$.

Por fim, os resíduos recicláveis representaram $27,4 \%$ da geração total de resíduos no RI no ano de 2009, com uma média de 16,2g/refeição. Em Carneiro et al. (2010), encontra-se um percentual de 10\% para a geração dos resíduos inorgânicos. Já no trabalho de Lafuente Junior (2012), os resíduos provenientes do estoque e armazenamento de um restaurante comercial corresponderam a aproximadamente $37 \%$ da geração total dos resíduos. Ao passo em que em Picciafuoco (2013) os resíduos recicláveis corresponderam a apenas $1 \%$ da geração total de resíduos.

Embora se considere importante comparar com valores estipulados na literatura, Abreu (2003 apud Augustini et al., 2008) afirmam que a avaliação da redução e o aumento dos resíduos gerados deve ser 
sempre comparativa dentro da própria unidade, permitindo-se acompanhar a evolução da geração de resíduos ao longo do tempo. Neste estudo, a geração dos resíduos foi acompanhada durante o período de um ano, permitindo visualizar o caminho da geração de resíduos sólidos dentro do RI em todos os seus setores.

\section{CONSIDERAÇÕES FINAIS}

Os dados foram coletados durante o período de um ano e possibilitaram um método para determinação de índices de geração de resíduos sólidos e controle de sobras de alimentos em restaurantes industriais de grande porte. A proposta metodológica adotada pode contribuir para aprimorar as práticas desenvolvidas em RIs, uma vez que na literatura, os estudos da área se restringem-se a coleta de dados de no máximo um mês.

Conclui-se que é notória elevada a quantidade de resíduos orgânicos gerados, indicando que as etapas de retorno das bandejas, pré-preparo e sobra limpa são as que devem ser priorizadas para a minimização da geração de resíduos no RI estudado. A modificação de cardápios através da substituição de alimentos que produzem menor quantidade de resíduos, a utilização de produtos pré-processados e a aplicação do processo de compostagem, aliados à educação ambiental dos funcionados e dos consumidores, são fatores importantes para modificar este cenário.

No entanto, ressalta-se que raramente um restaurante produz exatamente as quantidades de alimentos que serão consumidas pelos usuários. Consequentemente, a sobra limpa é uma fonte geradora de resíduos mesmo que sejam aplicados os procedimentos corretos exigidos pela legislação vigente.

\section{REFERÊNCIAS}

ABERC. Mercado Real. Associação Brasileira das Empresas de Refeições Coletivas. 2008. Disponível em: <http://www.aberc.com.br/mercadoreal.asp?IDMenu=21>. Acesso em: 20 mar. 2014.

ABNT. ABNT NBR 1004. 2. ed. 2004. Disponível em: < http://www.aslaa.com.br/legislacoes/NBR\%20n\%20100042004.pdf>. Acesso em: 15 jul. 2014.

ABRELPE. Panorama dos resíduos sólidos no Brasil. 116 f. 2012.

AUGUSTINI, V. C. M.; KISHIMOTO, P.; TESCARO, T. C.; ALMEIDA, F. Q. A. de. Avaliação do índice de resto-ingesta e sobras em unidade de alimentação e nutrição (UAN) de uma empresa metalúrgica na cidade de Piracicaba/SP. SimbioLogias, v. 1, n. 1, p. 99 - 110. Mai. 2008.

BILCK, A. P.; SILVA, D. L. D. da; COSTA, G A. N.; BENASSI, V. T.; GARCIA, S. Aproveitamento de subprodutos: restaurantes de Londrina. Revista em Agronegócio e Meio Ambiente, v.2, n.1, p. 87 - 104, Jan. - Abr. 2009

BRASIL. Decreto $n^{\circ}$ 7.404. 2010. Disponível em: < http://www.planalto.gov.br/ccivil_03/_ato20072010/2010/Decreto/D7404.htm>. Acesso em: 15 jul. 2014.

CARNEIRO, C. M. L.; LIMA, A. M. de; AZEVEDO, J. B. de; CASTRO, M. G.; SILVA, K. M. B. da. Diagnóstico dos resíduos sólidos produzidos no restaurante universitário da UFRN. In: ENCONTRO NACIONAL DE ENGENHARIA DE PRODUÇÃO, 30., 2010, São Carlos. Maturidade e desafios da Engenharia de Produção: competitividade das empresas, condições de trabalho, meio ambiente... São Carlos: 2010. p. 1 - 13.

CASTRO, M. C. A. A. A política nacional de resíduos sólidos e os planos municipais de gestão integrada. In: CONGRESSO DE INICIAÇÃO CIENTIFICA, 8., 2013, Araraquara. Anais VIII Congresso de Iniciação Cientifica... Araraquara: Uniara, 2013, v. 1, p. $26-32$.

CORRÊA, M. S.; LANGE, L. C. Gestão de resíduos sólidos no setor de refeição coletiva. Pretexto, Belo Horizonte, v. 12 , n. 1 , p. $29-54$, Jan. - Mar. 2010.

COSTA, F. X.; LUCENA, A. M. A.; TRESENA, N. L.; GUIMARÃES, F. S.; GUIMARÃES, M. B.; SILVA, M. M. P. da; GUERRA, H. O. C. Estudo qualitativo e quantitativo dos resíduos sólidos do Campus I da Universidade Estadual da Paraíba. Revista de Biologia e Ciências da Terra, v. 4, n. 2. 2004. 
LAFUENTE JUNIOR, A. N. A. Resíduos sólidos em restaurantes comerciais: um estudo de caso na cidade de Santos/SP. Revista de Tecnologia Aplicada, v. 6, n. 2, p. 44 - 61. 2012.

LOPES, M. L.; FONSECA, V. V. Estudo do manejo dos resíduos de um restaurante institucional da região sul fluminense. Interbio, v. 7, n. 1, p. $47-53.2013$.

MENEZES, R. L.; SANTOS, F. C. A.; LEME, P. C. S. Projeto de minimização de resíduos sólidos no restaurante central do campus de São Carlos da Universidade de São Paulo. In: ENCONTRO NACIONAL DE ENGENHARIA DE PRODUÇÃO, 22., Curitiba. 2002. p. $1-8$

MONTE, C. R. V. do; COSTA, V. S. R. da; COUTO, S. R. M.; LANZILLOTTI, H. S. Aplicação de um modelo para avaliar projetos de unidades de alimentação e nutrição. Nutrição Brasil, v. 3, n. 1, p. 11 - 17. 2004.

PERUCHIN, B.; GUIDONI, L. L. C.; CORRÊA, L. B.; CORRÊA, E. K. Gestão de resíduos sólidos em restaurante escola. Tecno-lógica, Santa Cruz do Sul, v. 17, n. 1, p. 13 - 23, Jan. - Jun. 2013.

PICCIAFUOCO, Beatriz Di Francesco. Proposta de compostagem para os resíduos sólidos orgânicos do restaurante universitário da Unesp de Rio Claro. 2013. Trabalho de Conclusão de Curso (Graduação em Engenharia Ambiental) Instituto de Geociências e Ciências Exatas, Universidade Estadual Júlio de Mesquita Filho, Rio Claro, 2013.

PICCIAFUOCO, B. F.; TAKEDA, C. M.; SOUZA, L. A.; CASTRO, M. C. A. A. A. Quantificação dos resíduos sólidos gerados no restaurante universitário do Campus de Rio Claro-UNESP: avaliação do potencial de compostagem. In: WORKSHOP DE GESTÃO DE RESÍDUOS DA UNESP, 3., 2014, Araçatuba.

SIQUEIRA, L. C. Produção de resíduos no restaurante universitário: Diagnóstico para ações de educação ambiental no programa agenda 21 da universidade de Brasília. 2002. 128 f. Dissertação (Mestrado em Ecologia) - Instituto de Ciências Biológicas, Universidade de Brasília, Brasília, 2002.

SOUZA, M. F.; FAGUNDES, A. K.; MILANI, I. C. B.; NEBEL, A. L. C.; TAVARES, V. E. Q.; SUZUKI, L. E. A. S.; COLLARES, G. L. Caracterização dos resíduos sólidos gerados em um restaurante universitário. In: ENCONTRO DE PÓSGRADUAÇÃO, 11., Pelotas, 2010.

THIAGARAJAH, K.; GETTY, V. M. Impact on plate waste of switching from a tray to a trayless delivery system in a university dining hall and employee response to the switch. Journal of the academy of nutrition and dietetics, v. 113 , p. 141 $-145.2013$.

TRANCOSO, S. C.; TOMASIAK, F. S. Estruturação de uma unidade de alimentação e nutrição. Nutrição Brasil, Rio de Janeiro, v. 3, n. 1, p. $18-21$, Jan. - Fev. 2004.

VIDMANTAS, J. B. D.; TIRLONE, C. A. S.; LOUREIRO, P.; NASCIMENTO, R. A. do. Perfil do gerenciamento de resíduos alimentares dos FastFood do shopping da cidade Dourado/MS. In: SIMPÓSIO INTERCÂMBIO BRASIL-JAPÃO EM SUSTENTABILIDADE, 3., 2010, Campo Grande. p. 1 - 6. 